\section{Timing of puberty — body size or reproductive optimization?}

\author{
Yehuda Limony (1D and Slawomir Koziel
}

In the July 2020 issue of Nature Reviews Endocrinology Ben Bar-Sadeh and colleagues reviewed the up-to-date knowledge about early childhood epigenetic changes of the reproductive system in response to environmental events (Bar-Sadeh, B. et al. Unravelling the role of epigenetics in reproductive adaptations to early-life environment. Nat. Rev. Endocrinol. 16, 519-533 $(2020))^{1}$. One of the changes described is the change in the time of onset of puberty. This change is described by the authors as adaptive change, part of multiple adaptation changes in the reproductive system, to "optimize success."

We would like to add another point of view regarding this change. We think that the change in the timing of puberty is aimed primarily at achieving target height and so at optimizing body size. We have reached this conclusion in our recently published auxological study based on two cohorts: Israeli and Polish ${ }^{2}$. In our study, we found a correlation between the timing of puberty and the child's target height (calculated as the average of normalized parental heights) and described it in a mathematical model. We found that children who grow in childhood along a height percentile higher than their target height percentile (a situation termed by us as positive height gap) will start puberty earlier than average. Children who grow along a height percentile shorter than their target height percentile (termed negative height gap) will start puberty later than average. When a pubertal growth spurt starts earlier than average, the final height percentile is lower than the height percentile at its start ${ }^{3}$. Thus, whenever early puberty accompanies a positive height gap, it narrows the height gap at the end of the pubertal growth spurt, resulting in a final height closer to the target height (that is, body size similar to that of parents). It is therefore probable that this association between the height gap and timing of puberty is part of the targeted process of growth that might have an evolutionary advantage.

In their article, Bar-Sadeh and colleagues cite studies that demonstrated the advancement of the timing of puberty in immigrant populations. Indeed, as shown in many studies, the risk of precocious puberty is greater in children who have been adopted or immigrated with a relocation from a developing country to a developed one $^{4,5}$. It should be noted, however, that the advancement of the age of puberty is preceded by a period of accelerated growth and the greater this catch-up growth, the more advanced is the timing of puberty ${ }^{6}$. It is possible that the catch-up growth created a positive height gap, but unfortunately, these studies lack data on parental height.

We agree with Bar-Sadeh and colleagues who emphasize the need for multifaceted approaches to elucidate this intriguing aspect of human biology. However, in our opinion, research on the possible association between the timing of puberty and epigenetic changes should also be directed to genes associated with height growth.
There is a reply to this letter by Bentley et al. Reply to: Timing of puberty - body size or reproductive optimization? Nat. Rev. Endocrinol. https://doi.org/10.1038/s41574021-00502-4 (2021).

Yehuda Limony iD ${ }^{1 凶}$ and Slawomir Koziel ${ }^{2}$ 'Clalit Health Services, Community Medicine, Migdaley-Kenyon-Hanegev, Be'er-Sheva, Israel. ${ }^{2}$ Hirszfeld Institute of Immunology and Experimental Therapy, Wrocław, Poland.

凶e-mail: limony@zahav.net.il

https://doi.org/10.1038/s41574-021-00501-5

1. Bar-Sadeh, B. et al. Unravelling the role of epigenetics in reproductive adaptations to early-life environment. Nat. Rev. Endocrinol. 16, 519-533 (2020).

2. Limony, Y., Koziel, S. \& Friger, M. Association between the onset age of puberty and parental height. PLOS ONE 14, e0211334 (2019).

3. Limony, Y., Koziel, S. \& Friger, M. Age of onset of a normally timed pubertal growth spurt affects the final height of children. Pediatr. Res. 78, 351-355 (2015).

4. Teilmann, G., Pedersen, C. B., Skakkebaek, N. E. $\&$ Jensen, T. K. Increased risk of precocious puberty in internationally adopted children in Denmark. Pediatrics 118, e391-399 (2006).

5. Virdis, R. et al. Precocious puberty in girls adopted from developing countries. Arch. Dis. Child. 78, 152-154 (1998)

6. Proos, L. A. Growth $\bar{\alpha}$ development of Indian children adopted in Sweden. Indian J. Med. Res. 130, 646-650 (2009)

\section{Competing interests}

The authors declare no competing interests.

\title{
Reply to: Timing of puberty - body size or reproductive optimization?
}

\section{Gillian R. Bentley (D), Barry A. Bogin (D), Ben Bar-Sadeh, Reinhard Stöger(D) and Philippa Melamed (1)}

We appreciate the comments by Limony and Koziel on our Review (Bar-Sadeh, B. et al. Unravelling the role of epigenetics in reproductive adaptations to early-life environment. Nat. Rev. Endocrinol. 16, 519-533 (2020) $)^{1}$ in suggesting that pubertal development might be related to achieving a target height (Limony, Y. \& Koziel, S. Timing of puberty - body size or reproductive optimization? Nat. Rev. Endocrinol. https:// doi.org/10.1038/s41574-021-00501-5 (2021))2.

The authors state that: "the change in the timing of puberty is aimed primarily at achieving target height and so at optimizing body size." Our approach is taken from the perspective of life history theory, which argues that energy availability is allocated between growth, maintenance and reproduction, and that trade-offs exist to optimize Darwinian fitness as in reproductive success ${ }^{3,4}$. However, in their correspondence, Limony and Koziel suggest that height is the currency of optimization in the process of pubertal maturation as opposed to fitness. We would argue instead that changes in the timing of puberty reflect endocrine responses to available energy, and that adjustments to that availability are reflected in the plasticity, facilitated by epigenetic mechanisms that influence reaction norms. This viewpoint is supported in animal studies, as noted in our review ${ }^{1}$; we find it difficult to reconcile how Limony's theory regarding target height translates across the animal kingdom.

We read Limony et al.s earlier article in which they described an association between height gap (that is, difference between the actual height and the genetically determined 'target height') and age at onset of puberty ${ }^{5}$, and which appears to provide the basis for their Correspondence. We would respectfully point out that James Tanner described and analysed 'target heights' and catch-up growth in his Nature article nearly 60 years ago, including relevance to the timing of pubertal onset ${ }^{6}$. Moreover, the connection between the onset of the adolescent growth spurt and height were documented in the 